DRAFT VERSION SEPTEMBER 4, 2018

Preprint typeset using LTEX style emulateapj v. 5/2/11

\title{
CHARACTERIZING THE BROWN DWARF FORMATION CHANNELS FROM THE INITIAL MASS FUNCTION AND BINARY-STAR DYNAMICS
}

\author{
Ingo Thies $^{1}$, Jan Pflamm-Altenburg ${ }^{1}$, Pavel Kroupa ${ }^{1}$, Michael Marks ${ }^{1}$ \\ Draft version September 4, 2018
}

\begin{abstract}
The stellar initial mass function (IMF) is a key property of stellar populations. There is growing evidence that the classical star-formation mechanism by the direct cloud fragmentation process has difficulties reproducing the observed abundance and binary properties of brown dwarfs and very-low-mass stars. In particular, recent analytical derivations of the stellar IMF exhibit a deficit of brown dwarfs compared to observational data. Here we derive the residual mass function of brown dwarfs as an empirical measure of the brown dwarf deficiency in recent star-formation models with respect to observations and show that it is compatible with the substellar part of the Thies-Kroupa IMF and the mass function obtained by numerical simulations. We conclude that the existing models may be further improved by including a substellar correction term that accounts for additional formation channels like disk or filament fragmentation. The term "peripheral fragmentation" is introduced here for such additional formation channels. In addition, we present an updated analytical model of stellar and substellar binarity. The resulting binary fraction and the dynamically evolved companion mass-ratio distribution are in good agreement with observational data on stellar and very-low-mass binaries in the Galactic field, in clusters, and in dynamically unprocessed groups of stars if all stars form as binaries with stellar companions. Cautionary notes are given on the proper analysis of mass functions and the companion mass-ratio distribution and the interpretation of the results. The existence of accretion disks around young brown dwarfs does not imply that these form just like stars in direct fragmentation.
\end{abstract}

Subject headings: binaries: general — brown dwarfs — methods: numerical — methods: statistical — stars: low-mass - stars: luminosity function, mass function -

\section{INTRODUCTION}

The stellar initial mass function (IMF) is a key tool for star-formation research because it mirrors the processes of the formation of stellar populations (Bastian et al. 2010, Kroupa et al. 2013). Consequently, the IMF has been subject to extensive research both observationally and theoretically. In recent years the majority of the star-formation community has favored the assumption of continuous star formation from the lowest-mass brown dwarfs (BDs) to the most massive stars (Padoan \& Nordlund 2002, 2004, Hennebelle \& Chabrier 2008). However, there is evidence for at least two separate formation channels for most BDs on the one hand and most stars on the other. For instance, careful analysis of the observational data reveals a disagreement between the theoretical predictions of the binary separation distributions of BDs and stars and the observed ones (Bouy et al. 2003, Burgasser et al. 2003, Martín et al. 2003 and Close et al. 2003). In this light the key assumption of a uniform or continuous formation mechanism assumed in most star-formation models needs to be questioned. Based on observational data and N-body computations, Parker \& Goodwin (2011) conclude that the birth population of very-low-mass binaries with system masses below $0.2 M_{\odot}$ must be very different from that of M-dwarfs. Another important issue is the "BD desert" (McCarthy, Zuckerman, \& Becklin 2003), an observed dearth of BD companions to stars. Unlike earlier studies like e.g. by Grether \& Lineweaver (2006) who interpret this as being related to the companion mass ratio rather than the absolute mass, the more recent survey by Dieterich et al. (2012) deduce a lower absolute mass limit of companions to stars close to $0.1 \mathrm{M}_{\odot}$.

\footnotetext{
${ }^{1}$ Helmholtz-Institut für Strahlen- und Kernphysik (HISKP), Universität Bonn, Nussallee 14-16, D-53115, Germany
}

Analytical approaches by Padoan \& Nordlund (2002. PN02, from here) and Hennebelle \& Chabrier (2008, 2009 $\mathrm{HC} 08$, and $\mathrm{HC} 09$, hereafter) deduces the IMF from an analytical description of the distribution of prestellar cloud cores. While the stellar IMF is reproduced by these approaches, a significant deficit of BDs and very-low-mass stars (VLMSs, between about 0.08 and $0.2 M_{\odot}$ ) with respect to observationally constrained IMF descriptions (Kroupa 2001, Chabrier 2005. Thies \& Kroupa 2007, 2008, the latter three from here on referred to as C05, TK07, and TK08, respectively, and Kroupa et al. 2013) appears if realistic properties of the prestellar clouds are assumed. The fundamental reason why direct fragmentation of a turbulent molecular cloud rarely produces BDs is that the formation of a BD requires a highdensity gravitationally self-bound but very low mass fluctuation that cannot draw significant amounts of additional mass from an accretion reservoir (see also Adams \& Fatuzzo 1996). However, $\mathrm{HCO} 8$ and $\mathrm{HCO} 9$ speculate that this deficit might be solved by a refinement of their models by including turbulent fragmentation descriptions. A different interpretation is that their model is overall correct but reveals that an additional formation channel is required to match the observations. The IMF by $\mathrm{C} 05$ is an empirical and almost equivalent update of the IMF by Chabrier (2003). Fully hydrodynamical computations of whole star-forming clouds by e.g. Bate et al. (2003) and Bate (2009a) reproduce the formation of BDs largely from fragmenting circumstellar disks, although these simulations tend to overproduce BDs unless radiative heating is included (Bate 2009b, 2012).

In this paper we introduce the residual mass function (RMF) as a correction term for this BD deficiency in the analytical approaches by $\mathrm{PN} 02$ and $\mathrm{HC} 08$ with respect to the observation-based IMFs in C05 and TK07. In Section 2 the 
model is described and the RMF is defined. The results are presented in Section 3. followed by an analysis of the companion mass-ratio distribution in the overlap region of these two populations and a discussion of the BD desert and its claimed consistency with empirically determined IMF models (Reggiani \& Meyer 2011), in Section 4 In addition, the term peripheral fragmentation is introduced as the main formation channel of BDs.

\section{METHOD}

The basic idea is to quantify the deficit of analytically derived mass functions with respect to observationally constrained IMFs. The resulting deficit defines the RMF.

\subsection{Observational IMF models}

The stellar IMF is based on an extensive analysis of observational data from young stellar clusters and in the Galactic field (Kroupa et al. 1993, Kroupa 2001). Because the majority of multiple systems remain unresolved such IMFs need to be interpreted as system mass functions unless a careful numerical binary correction is used.

The empirical IMF by C05 used here is a system IMF based on the earlier IMF by Chabrier (2003), assuming BDs and stars forming the same way, thereby simply occupying different regions in the same continuous mass function, an assumption also made in the past by Kroupa (2001, 2002) TK07, on the other hand, provide an individual-body IMF that has been transformed into the corresponding system IMF by Monte Carlo random pairing among the star-like and the separate $B D$-like population introduced in TK07. This accounts for the observational evidence for two separate (albeit related) formation channels. In particular, there is a lack of observed BD companions to stars, especially for small separations (McCarthy et al. 2003. Grether \& Lineweaver 2006), whereas the statistical properties of binary BDs differ largely from stellar ones (Bouy et al. 2003, Burgasser et al. 2003. Martín et al. 2003. Close et al. 2003). As argued in Thies \& Kroupa (2007), the different binary properties of BDs and VLMSs on the one hand, and stars on the other, consequently suggest the existence of two separate formation channels. This thus leads to the requirement for two separate IMFs, each corrected for unresolved binaries, to describe the real individual-body mass function of a star-forming event.

These IMF descriptions, however, are purely observationally motivated and therefore do not explain the underlying physical processes leading to the actual mass spectrum.

\subsection{Analytical IMF models}

In recent years, several attempts have been made to understand the physics behind star formation and to reproduce its outcome. This section deals with the analytical models by PNO2 and HC08. In both PNO2 and HC08 the prestellar clump mass function is described by an analytical function with the Jeans mass, the length scale of the initial inhomogeneities and the Mach number, $\mathcal{M}$, as the main parameters (see Equation (24) in PN02 and Equation (44) in HC08). Both studies derive the IMF from the mass distribution of gravitationally unstable clumps based on empirical power spectra of turbulent flows within the molecular clouds. While the particular formalism differs in PN02 and HC08, fragmentation due to the supersonic interaction of gas sheets is the engine for forming Jeans-unstable clumps in both models. The resulting prestellar clump mass function is then transformed into a stellar IMF assuming a star-formation efficiency of $30 \%-50 \%$. The models have been tested in the range $3 \leq \mathcal{M} \leq 20$. In this paper, we restrict the model to the Mach number $\mathcal{M}=12$ in the case of $\mathrm{HC} 08$ (Figure 5 top therein) and $\mathcal{M}=10$ in PN02 because these Mach numbers are suggested as the most likely values in these papers (see p. 873 in PN02 and p. 406 in HC08). With these values both models match each other well in the stellar regime despite their different formulations. Higher Mach numbers would mainly shift the mass spectrum toward lower masses and decrease the MF toward the high-mass end. As shown in Figure 1 the PNO2 $\mathcal{M}=10$ mass function (longdashed curve) and the $\mathcal{M}=12 \mathrm{HC} 08$ mass function (solid curve) closely match in the stellar regime but are slightly different at the low-mass end. The resulting mass function deviates significantly from the observed stellar+substellar mass function in the substellar mass regime, as shown in Figures 2 and 3 for both theoretical models compared to the observationally constrained IMFs by $\mathrm{C} 05$ and TK07. To quantify this difference the model IMF, either $\mathrm{HC} 08$ or PN02, is subtracted from an observational reference mass function that is taken from $\mathrm{C} 05$ and TK07 in order to determine two estimates of the RMF: $\xi_{\text {res }}$,

$$
\xi_{\text {res }}(m)=\xi_{\text {obs }}(m)-\xi_{\text {theo }}(m),
$$

where $\xi_{\text {theo }}$ is any of the theoretical IMFs and $\xi_{\text {obs }}$ refers to any of the observationally constrained IMFs. In general, a mass function $\xi$ is defined as the differential number $N$ over the differential object mass $m$ :

$$
\xi(m)=\frac{\mathrm{d} N}{\mathrm{~d} m},
$$

and, in the logarithmic scale

$$
\xi_{\mathrm{L}}(m)=\frac{\mathrm{d} N}{\mathrm{~d} \log _{10} m / M_{\odot}}=(\ln 10) m / M_{\odot} \xi(m) .
$$

The TK07 IMF is the canonical IMF (Kroupa et al. 2013) that takes into account that BDs and some VLMSs need to be added as an additional population called $B D$-like, whereas most stars belong to the star-like population:

$$
\begin{aligned}
& \xi_{\mathrm{BD}}(m)=\mathcal{R}_{\text {pop }} k\left(\frac{m}{0.07}\right)^{-0.3}, 0.01<m \lesssim 0.15, \\
& \xi_{\text {star }}(m)=k\left\{\begin{array}{ll}
\left(\frac{m}{0.07}\right)^{-1.3}, & 0.07<m \leq 0.5, \\
k_{m}\left(\frac{m}{0.5}\right)^{-2.3}, & 0.5<m \leq m_{\max }
\end{array},\right.
\end{aligned}
$$

where $\mathcal{R}_{\text {pop }}$ is the $\mathrm{BD}$-like to star-like population ratio $\left(\mathcal{R}_{\text {pop }} \approx 0.2\right.$, TK07) and $k_{m}=\left(\frac{0.5}{0.07}\right)^{-1.3}$ ensures continuity of the stellar IMF at $0.5 M_{\odot}$. Here, $m_{\max }$ follows from the $m_{\mathrm{max}}-M_{\text {ecl }}$ relation (Weidner et al. 2010, 2013, Equation (10) in Pflamm-Altenburg et al. 2007) and approaches $150 M_{\odot}$ for the most massive clusters. Note the overlapping mass ranges of the populations indicating that bodies between about 0.07 and $0.15 M_{\odot}$ may belong either to the star-like or the BD-like population.

At the high-mass end of the BD-like population, the sharp truncation used in TK07 has been replaced in this study by a steep power-law function to reduce numerical artifacts in the sum IMF. We chose a power-law exponent of 10 to keep the effect on the BD-like to star-like ratio negligibly small. There is no such declining function applied to the star-like population that is intrinsically smoother due to the dynamical population synthesis (DPS) method described in Section 2.3 


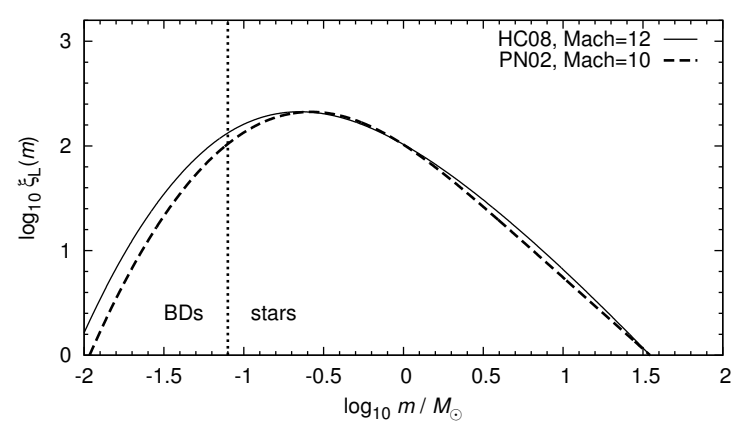

FIG. 1.- Comparison of the analytical system IMF by PN02 for $\mathcal{M}=10$ (their preferred value, dashed curve) with that by $\operatorname{HC} 08(\mathcal{M}=12$, solid curve). Both functions are scaled in this plot for equal peak height for comparison only. Although they nearly match in the stellar regime there are slight deviations in the substellar regime as well as in the positions (i.e. masses) of the peak.

In addition to this, star-like objects below $0.06 M_{\odot}$ and BDlike ones above $0.3 M_{\odot}$ are not considered. It should be noted here that the theoretical mass function obtained by Thies et al. (2010) from smoothed particle hydrodynamics (SPH) simulations also shows a steep decline above $0.1 M_{\odot}$ rather than an exact truncation.

Since the C05 stellar IMF used by HC08 is a system mass function rather than an individual-body-mass function, the TK07 canonical IMF has also been transformed into the corresponding TK07 system mass function using the Monte-Carlo model described in Section 2.3. The stellar component of the canonical TK07 system IMF is normalized to the C05 system IMF in the whole mass regime $\left(0.01-150 M_{\odot}\right)$, i.e.

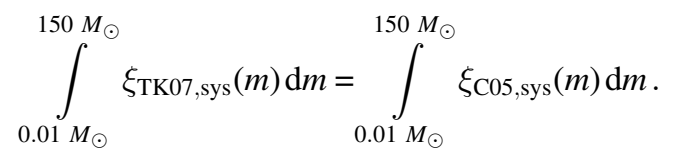

Here it is assumed that in the C05 IMF stars and BDs share the same IMF without a discontinuity or overlap, so the mass regime above $0.08 M_{\odot}$ corresponds to the stellar component in the TK07 model.

The BD multiplicity fraction is only about 10\%-20\% (Bouy et al.2003, Close et al.2003, Martín et al.2003; Kraus, White, \& Hillenbrand 2006 and Law et al. 2008) and is assumed to be equal for both C05 and TK07 IMFs.

Further, it has to be noted here that the RMF is based on the mass range between 0.01 and about $0.3 M_{\odot}\left(\log _{10} m / M_{\odot}\right.$ between -2 and -0.5 ) only, because the residuals in the stellar regime are due to slightly different functional descriptions rather than being physically constrained and are therefore neglected here. The residuals between the observational stellar IMF fits of $\mathrm{C} 05$ and TK07 and the HC08 and PN02 models are of potential interest to later work, but they are not further considered in this paper.

\subsection{Monte-Carlo model of the binarity of stars and brown dwarfs}

Besides the mass function itself, the binarity as a function of the primary-star mass and the CMRD are also important characteristics of stellar populations. They are studied here using a Monte Carlo approach with star-like and BD-like objects drawn from separate IMFs.

The binarity or binary fraction, $f$, is defined as the ratio of the number of binary or higher-order systems, $N_{\text {bin }}$, to the total number of systems, $N_{\text {sys }}$. Here, the term system includes multiple systems and singles (their number being noted as $N_{\text {sng }}$ ) as well. Then

$$
f=\frac{N_{\text {bin }}}{N_{\text {sys }}}=\frac{N_{\text {bin }}}{N_{\text {sng }}+N_{\text {bin }}} .
$$

For the star-like population we apply the binary DPS method developed by Marks, Kroupa, \& Oh (2011) and Marks \& Kroupa (2011), hereafter referred to as dynamical or DPS pairing. In DPS the binary stars are formed in a population of embedded clusters within which they are dynamically processed to yield the Galactic disk stellar single-plusbinary population. An attractive feature of this theory is its underlying assumption of the universality of binary properties of late-type stars being consistent with observational data (Marks \& Kroupa 2012, Leigh et al. 2014). For initial binaries with intermediate to large separations the DPS pairing method applies random pairing $2^{2}$ below a primary mass of $5 M_{\odot}$ and ordered pairing (such that $q \geq 0.9$ ) above. Here, $q=m_{\text {comp }} / m_{\text {prim }} \leq 1$, where $m_{\text {comp }}$ is the companion mass and $m_{\text {prim }}$ is the mass of the primary star. This initial binary population is then altered by dynamical evolution. Close binaries with orbital periods below about 10 days undergo eigenevolution (Kroupa 1995) and tend to equalize the companion masses. Note that this eigenevolution term alters the very-low-mass end of the starlike IMF. For the purpose of this work, however these effects only play a negligible role. Here, the initial or primordial binary fraction is $100 \%$, i.e. it is assumed that all stars form in binaries. The final (after dynamical processing in the embedded cluster) overall binary fraction is about $40 \%$ (i.e. $f=0.4$ ) but varies as a function of the primary-star mass. For M-dwarfs, in particular, it is as low as $25 \%$ while G-dwarfs show about $56 \%$ binarity. The binary fraction approaches $90 \%$ for $\mathrm{O}$ stars. For the BDlike population we chose an overall binary fraction of $20 \%$ (i.e. $f=0.20$ ), in accordance with TK07, TK08. About half of the members of observed average stellar populations are binaries, most of them remaining unresolved in typical starcluster surveys. However, very young and likely dynamically unevolved populations like the Taurus-Auriga association exhibit almost $100 \%$ binarity (Kroupa et al. 2013, Duchêne \& Kraus 2013, Reipurth et al. 2014). The number of systems must not be confused with the number of individual bodies, $N_{\text {bod }}=N_{\text {sng }}+2 N_{\text {bin }}$. Since higher-order multiples are relatively rare (Goodwin \& Kroupa 2005) they are summarized within the binary population in this work, so the total number of bodies is

$$
N_{\text {bod }}=2 N_{\text {bin }}+N_{\text {sng }} .
$$

The CMRD describes the relative number of binaries as a function of the companion-to-primary mass ratio. Observations reveal a continuous decline of $f$ as a function of the primary-object mass which has been interpreted as a continuous transition from the stellar to the substellar regime (Joergens 2008, Kraus \& Hillenbrand 2012, but see Thies \& Kroupa 2008). There is also a shift towards more equal-mass binaries $(q=1)$ for VLMS and BDs (Dieterich et al. 2012). These properties of the stellar population are well reproduced by DPS such that the origin and properties of binary populations are well understood.

\footnotetext{
${ }^{2}$ Marks et al. (2014, in prep.) show that random pairing with subsequent dynamical processing does indeed reproduce at least the observed low-mass stellar population (see also Kroupa 1995).
} 
In this study we used a Monte Carlo approach with the TK07 IMF (Equation (4)), i.e., with two separate populations, $B D$-like and star-like, ranging from 0.01 to $0.15 M_{\odot}$ and from 0.06 to the maximum stellar mass of $150 M_{\odot}$, respectively. The slightly larger mass ranges compared to Equation (4) are due to the steep power-law decline added to the mass borders to reduce numerical artifacts. The masses are drawn randomly from each IMF where the relative normalization between both populations is simply obtained by the number of objects drawn from each IMF.

BD-like objects are assumed to form as single substellar cores some of which are subsequently paired to binaries within a dense dynamically preprocessed environment like a massive extended accretion disk (Stamatellos et al. 2007a Thies et al. 2010, Basu \& Vorobyov 2012). Similarly, stellar binaries are assumed to be assembled from individually formed and subsequently paired stars. After the individualbody populations have been drawn from the IMF, two different methods are used for assembling the binaries. For stars we apply the DPS pairing method mentioned above.

$\mathrm{BD}$ binaries, on the other hand, are created by first drawing two objects from their separate population characterized by $\xi_{\mathrm{BD}}$ (Equation 4 ) with the pairing probability $p_{\text {pair }}$ being determined by a power law,

$$
p_{\text {pair }}=\operatorname{const} q^{\gamma}
$$

following the power-law bias scheme used by Goodwin (2013) for the mass ratio distribution of binaries from second (i.e. binary-forming) fragmentation. Whereas the case $\gamma=0$ corresponds to random pairing from the IMF, $\gamma>0$ describes a biased pairing rule with an increasing pairing probability toward equal-mass components. The extreme case of perfect equal-mass pairing corresponds to $\gamma=\infty$ but is in disagreement with data from the Very-Low-Mass Binary Archive (VLMBA; Burgasser et al. 2007) which also contains a few unequal substellar binaries. In practice, first an array of BD-likes is generated by drawing randomly from $\xi_{\mathrm{BD}}$ (Equation 4). All subsequent pairings to make binaries are performed on the array only. Random pairing is performed by randomly drawing a companion from the array for each object of the same population. If the companion is more massive than the considered object it becomes the primary, and otherwise it is the secondary component. In the case of biased pairing an additional decision is made whether a companion randomly drawn from the population is accepted to be a partner or rejected, depending on the probability $p_{\text {pair }}$ in Equation (8). A rejected partner may later be chosen as a partner to another object. Objects that, on the other hand, are already bound in a binary are skipped in the pairing procedure henceforth. The biased pairing procedure is iterated until the required binary fraction $f$ is achieved. Because the objects are chosen in random order, this does not introduce any additional bias besides $p_{\text {pair }}$ to the binary populations. The method ensures that the slope of the canonical IMF is retained, in contrast to methods that select components according to a mass-ratio distribution only.

\section{RESULTS}

\subsection{Residual Mass function for Semianalytical Models}

In this section we present the RMFs obtained from the BD/VLMS deficit of the analytical IMF models by PN02 and $\mathrm{HC} 08$. The RMF is derived for a particular and, according to PNO2 and HC08, typical parameter set, in particular the tur- bulent Mach number. Other parameters yield different RMFs, so only the general functional shape for a likely parameter set is presented here. It is further shown that such an RMF provides a correction term to the stellar IMFs in order to fit a reasonable overall IMF without the BD deficit observed in the purely stellar ones. Figure 2 shows the results of our calculations for the analytical model from $\mathrm{HC} 08$ with the $\mathrm{C} 05$ and TK07 system IMF as the observational reference (upper and lower panels, respectively). In both cases the RMF is limited to the mass range below $0.3 M_{\odot}\left(\log _{10} m / M_{\odot}<-0.5\right)$ because the slightly different shapes of the IMFs in the stellar region are beyond the scope of this paper. The local minima near $\log _{10} m / M_{\odot}=-0.8$ (upper panel) and -1.4 (lower panel) occur because the difference between the HC08 mass function and the empirical mass function almost vanishes locally. This behavior is highly sensitive to the normalization of the IMFs and is most prominent in the case of the discontinuous TK07 IMF. Peaking near the hydrogen-burning mass limit the RMF declines steeply and effectively vanishes above $\log _{10} m / M_{\odot}=-0.5$. The peak mass, however, is better represented in the residual to TK07 in Figure 2 whereas the RMF from $\mathrm{C} 05$ appears to be shifted toward lower masses by about a factor of two (i.e. an offset of about -0.3 on the logarithmic scale).

In the case of the PN02 analytical model, as shown in Figure 3 , the result with respect to the C05 IMF covers a mass range similar to $\mathrm{HCO} 8$ but is also continuous. This is expected because both analytical models have a functional form similar to the C05 IMF, namely an extended log-normal-type shape. In the case of the TK07 system IMF (Figure 3) there is a prominent "dip" in the RMF around $\log _{10} m / M_{\odot}=-1.3$ (i.e. $m=0.05 M_{\odot}$ ). The reason is the near-equality of TK07 and PN02 at this point, which varies sensitively with the normalization of the TK07 system IMF and its substellar component. As with the HC08 model, the RMF of PN02 versus C05 peaks at lower masses by a factor of about two.

\subsection{Comparison with Simulation Data}

The RMF obtained in the previous section is compared here with the mass spectrum of substellar clumps formed in the SPH models of Thies et al. (2010). Therein the induced fragmentation of massive extended circumstellar disks due to perturbation by passing stars in an embedded cluster has been studied. Using an SPH code with a radiative cooling approximation (Stamatellos et al. 2007b) gravitational instabilities have been demonstrated to form through tidal perturbations and to form compact clumps with typical masses between 0.01 and $0.15 M_{\odot}$. The mass function of the thus-formed clumps, the "SPH mass function" (SPH MF) hereafter, has been shown in Thies et al. (2010) to be in agreement with the substellar component of the observed system IMF from TK07. In the current study, the SPH mass function is based on 80 objects formed in 29 computations, some of them performed in continuation of TK07.

In Figure 4 the (scaled) SPH mass function is compared to the corresponding RMF. The overall shape of the RMF essentially matches the SPH mass function which features a general increase with increasing mass, a peak around $0.1 M_{\odot}$ and a rapid decay for higher masses, thus characterizing a population that is restricted to the substellar and very-low-mass stellar regime. As mentioned by Thies et al. (2010), these results are quite similar to those in Stamatellos \& Whitworth (2009) who computed BD formation in self-fragmenting disks. Therefore, the results from triggered fragmentation can 


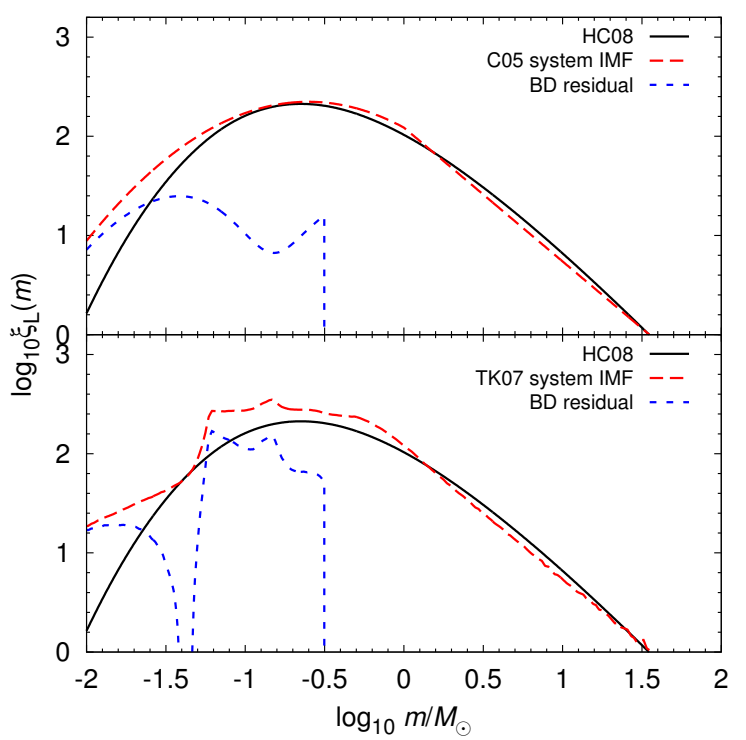

FIG. 2.- Upper panel: the analytical IMF model for $\mathcal{M}=12$ by $\mathrm{HC} 08$ (solid line) compared to the empirical IMF by Chabrier (2005) (dashed line). These functions, originally defined as system mass functions, have been normalized to match in the stellar regime. The dotted line represents the residual mass function, i.e. the difference between both mass functions. The gaps in the RMFs are caused by the intersection of the HC08 model IMF with the empirical IMFs. They are highly sensitive to the normalization of the IMFs. Lower panel: same as in the upper panel but with the combined bimodal IMF according to Thies \& Kroupa (2007) (dashed line). Note that the RMF is truncated for $\log _{10} m / M_{\odot} \geq-0.5$ because the different functional shapes of the stellar parts are not considered here.

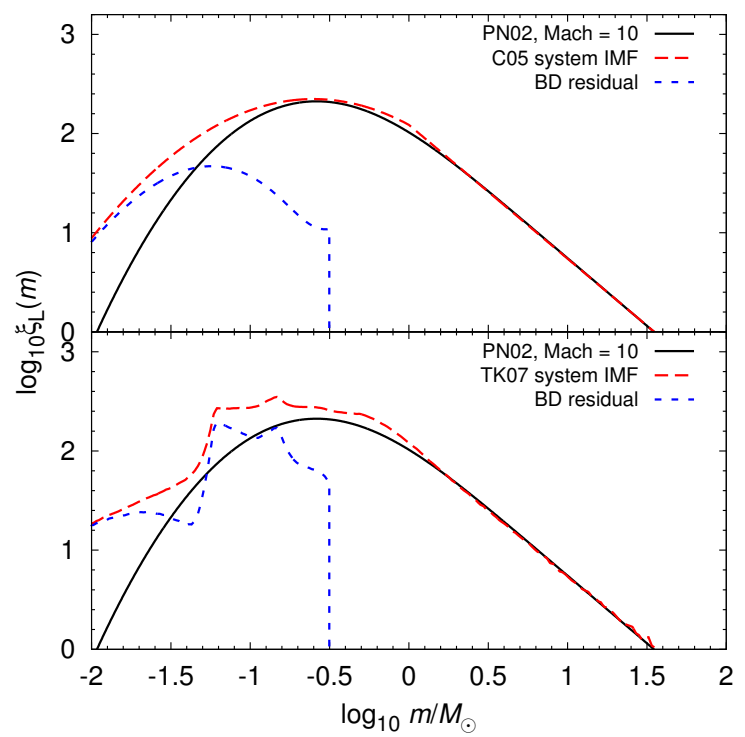

FIG. 3.- Upper panel: the same as in Figure 2 but with the clump mass function model by PNO2 instead of HC08. Lower panel: same as Figure 2 lower panel, but with the clump mass function model by PN02 instead of HC08. Here, the RMF does not contain any gaps.

be taken as representative for the general disk fragmentation outcome. Both scenarios assume that the substellar clumps formed in a fragmenting disk are ejected by either mutual dynamical interactions of clumps in the disk or in subsequent stellar encounters. According to Basu \& Vorobyov (2012) some of these clumps are disrupted by the tidal forces during the ejection or are destroyed because of migration onto the

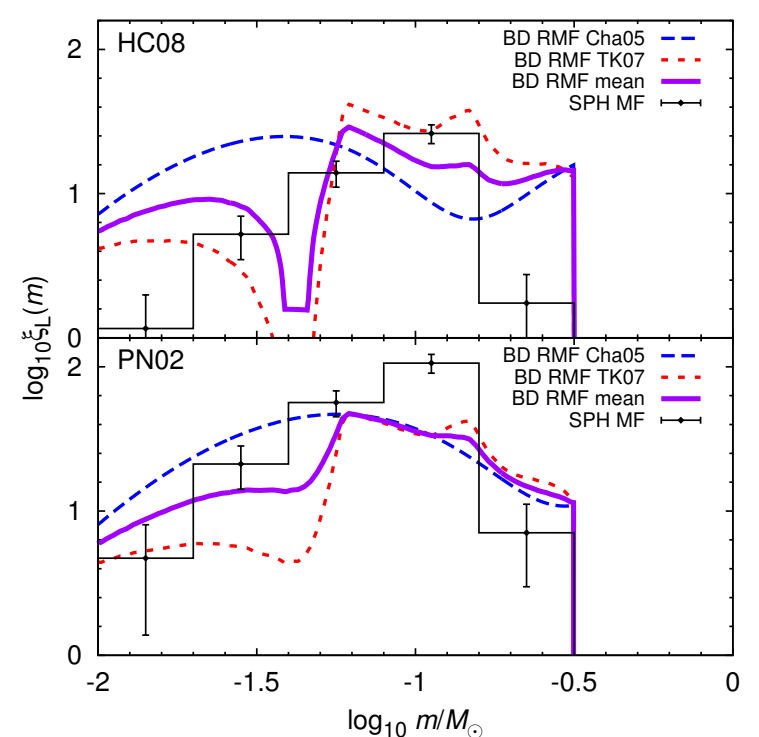

FIG. 4.- Residual mass functions of HC08 (upper panel, from Figure 2 and PNO2 (lower panel) with respect to the system mass functions by $\mathrm{C} 05$ (dashed line, from Figure 3 and TK07 (dotted line) as well as the average of both (solid line) in comparison to the SPH mass function from Thies et al. (2010 including post-population SPH data), shown as a histogram (normalized in $\log _{10} \xi_{L}$ to the RMF).

central star. The impact of this effect on the mass distribution will be subject to future research.

Given the uncertainties of the PNO2 and HC08 MFs the RMF based on them can be considered to be in agreement with the substellar IMF in both TK07 and Thies et al. (2010). Interestingly, the average of both RMFs (each weighted as $50 \%$ ) provides an even better fit to the SPH data (see the solid curves in Figure 4). Use of such an average may be motivated by the expectation that the true IMF below $\approx 0.2 M_{\odot}$ is likely between the TK07 and C05 quantifications.

\subsection{Monte Carlo Study Applied to Observations}

The IMF from the Monte Carlo study described in Section 2.3 is shown in Figure 5 in comparison to the system mass function (dash-dotted curve), the primary-body mass function (dashed curve) and the individual-body mass function (solid curve). The upper panel displays the sum IMF with both populations combined, as it would be seen by an observer. In the lower panel, both populations are plotted separately.

We note that Figure 12 in Kirk \& Myers (2012), which shows the observed average mass functions of young stellar clusters, groups and isolated objects across the stellarsubstellar boundary, reveals a prominent step near the stellarsubstellar border that is very similar to the one that emerges from our analysis as plotted in the upper panel of Figure 5 The system MF (dash-dotted curve) is to be compared with the mass function by Kirk \& Myers (2012). Because Kirk \& Myers (2012) only studied low-density, dynamically unevolved populations, the stellar system IMF is modelled here with a binary fraction of $80 \%$ (see also fig. 10 in Marks et al. 2011). For the Galactic field, for which the rest of our study has been performed, the binary fraction is only about $40-50 \%$ for low-mass stars.

Figure 6 depicts as the solid line the binarity as a function of the primary-body mass (i.e. the system mass in the case of single objects) from the DPS pairing model for the Galactic field population for embedded clusters with a half-mass radius 


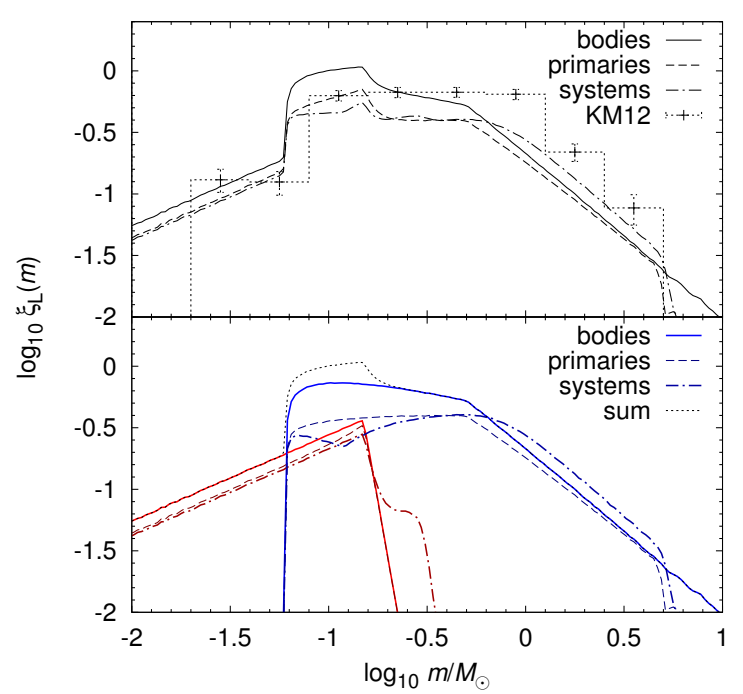

FIG. 5.- Upper panel: the combined IMF of BD-like and star-like objects from our Monte Carlo calculations for individual-body masses (solid curve), primary-body masses (dashed curve), and system masses (dash-dotted curve). The BD-like binarity is $20 \%$ here, and the star-like one is here about $80 \%$ on average, to match the case of dynamically unevolved populations (see text). For comparison, the histogram for grouped stars in Kirk \& Myers 2012 "KM12"), which also shows a discontinuity near the stellar-substellar border, is included as the dotted histogram. Lower panel: the IMFs for the BD-like and star-like populations plotted separately. The line patterns are the same as in the upper panel for each separate IMF. For comparison, the individual-body sum IMF is superimposed (thin dotted curve, the same as the solid curve in the top panel). Note that the very-low-mass end of the star-like IMF is slightly depleted due to eigenevolution (Marks et al. 2011).

of $0.1 \mathrm{pc}$ for the underlying cluster population. The longdashed line indicates the case of a half-mass radius of $0.2 \mathrm{pc}$ which yields an up to $20 \%$ higher multiplicity. These model Galactic field populations, computed with DPS (Section 2.3), assume that all stars form in binary systems in a population of embedded star clusters that evolve and disperse their stars into the field.

These two cases are not to be taken as statistically strict confidence limits but rather as two likely cases that have been studied in Marks \& Kroupa (2011), and the initial stellar binary population is indicated by the shaded region at the top.

The stellar binary fraction resulting from DPS is about $f=0.4$ on average in the low-mass region below $1 M_{\odot}$ for the case of $0.1 \mathrm{pc}$. The single-hatched region indicates the large uncertainty of the observed binarity in the substellar region. For comparison, Galactic-field observational data are overplotted for A stars (VAST survey, De Rosa et al.2014, asterisks), G and late-F stars (Duquennoy \& Mayor 1991, filled squares), $M$ to $G$ stars (Kroupa et al. 2003, open squares), M dwarfs (Fischer \& Marcy 1992; upright triangle), and L dwarfs (Reid et al. 2008; upside-down triangle).

Despite consisting of two separate populations (BD-like and star-like) the binary fraction declines continuously from the stellar toward the substellar regime. As discussed in Marks \& Kroupa (2011) and Marks \& Kroupa (2012), this is primarily due to the dynamical processing of the star-like population. In part, the mass overlap of the star-like and the BD-like population also contributes to this transition. It remains to be shown in future work that the apparent observed trend toward narrow binary separation distributions for latetype $\mathrm{M}$ dwarfs reported by Janson et al. (2014) can also be reproduced with DPS by this overlap.

\subsection{Binarity and Companion Mass-ratio Distribution}

The observable BD CMRD, which is the CMRD for all primaries (BD-like and star-like) below $0.08 M_{\odot}$, is shown in Figure 7. As can be seen the case of strongly biased pairing with $\gamma=2$ (solid line) matches the measured data from the VLMBA better (and also see Dieterich et al. 2012) than the random-pairing $q(\gamma=0$, dotted line $)$ and weakly biased pairing models $(\gamma=1$, dashed line $)$. In addition, the case $\gamma=4$ (Duchêne \& Kraus 2013; dash-dotted line) is shown. The binarity of BDs near the hydrogen-burning mass limit of $0.08 M_{\odot}$ is quite low in the model, only about $11 \%-12 \%$ which is largely due to the "dip" in the binarity in the VLMS region of the DPS pairing method (see Figure 6). This local minimum is a direct consequence of the two overlapping populations. Reid et al. (2008) indeed report a similar binary fraction of $12.5_{-3}^{+5} \%$ for L dwarfs. If a local minimum is confirmed by future surveys with sufficient precision in mass this would further support an overlap of two separate populations in this mass region. However, in the current data this feature is within the uncertainties of the observational data.

\subsection{Contribution of Peripheral Fragmentation to BDs}

One important implication of the model discussed in this paper is the hybrid nature of BDs and VLMSs. Although there is a minor contribution by the star-like population from direct fragmentation, the majority of BDs are contributed by the BD-like population through dynamically preprocessed gas (e.g. fragmenting circumstellar disks (Stamatellos \& Whitworth 2009, Thies et al. 2010). Because this preprocessed material often occurs in the peripheral regions of star formation (e.g. in the outer parts of disks) we propose the term peripheral fragmentation for the $\mathrm{BD}$-like formation channel. Of all BDs between 0.01 and $0.08 M_{\odot} 64 \%$ are contributed by the BD-like population, and $19 \%$ of $\mathrm{M}$ dwarfs between 0.08 and $0.45 M_{\odot}$ are BD-like. This result, however, is highly sensitive to the chosen lower mass border of the star-like regime. Here, we assumed it to be $0.06 M_{\odot}$. If, on the other hand, a sharp truncation of the star-like regime at $0.08 M_{\odot}$ is chosen, the BD-like fraction of BDs is $100 \%$ but still $18 \%$ of M dwarfs are BD-like. Because star-like BDs and VLMSs may be detectable by larger circumsubstellar disks or, if applicable, wide binary separations, future high-resolution observations will help to further constrain the BD-like and star-like mass borders.

\section{DISCUSSION}

In this paper we have introduced the RMF as a correction term for the analytical star-formation models by PNO2 and $\mathrm{HC} 08$ to match the observationally constrained IMF by Chabrier (2005) and Thies \& Kroupa (2007). The effective deficit of BDs and VLMSs in these theoretical models with respect to the observed BD statistics suggests the requirement for additional formation channels in these analytical IMF models. HC08 speculate that this deficit, being at least at the edge of significance (see uncertainties shown in Figure 4-23 in Kroupa et al. 2013), may be solvable by an improved algorithm that accounts for the effects of turbulence and other dynamical processing of the prestellar gas. The expected contribution of these additional effects to the IMF may be understood as a separate population or, at least, as an additional formation channel of BDs and VLMSs and can formally be described by an additional correction term. It is also applicable as a test for future star-formation models. 


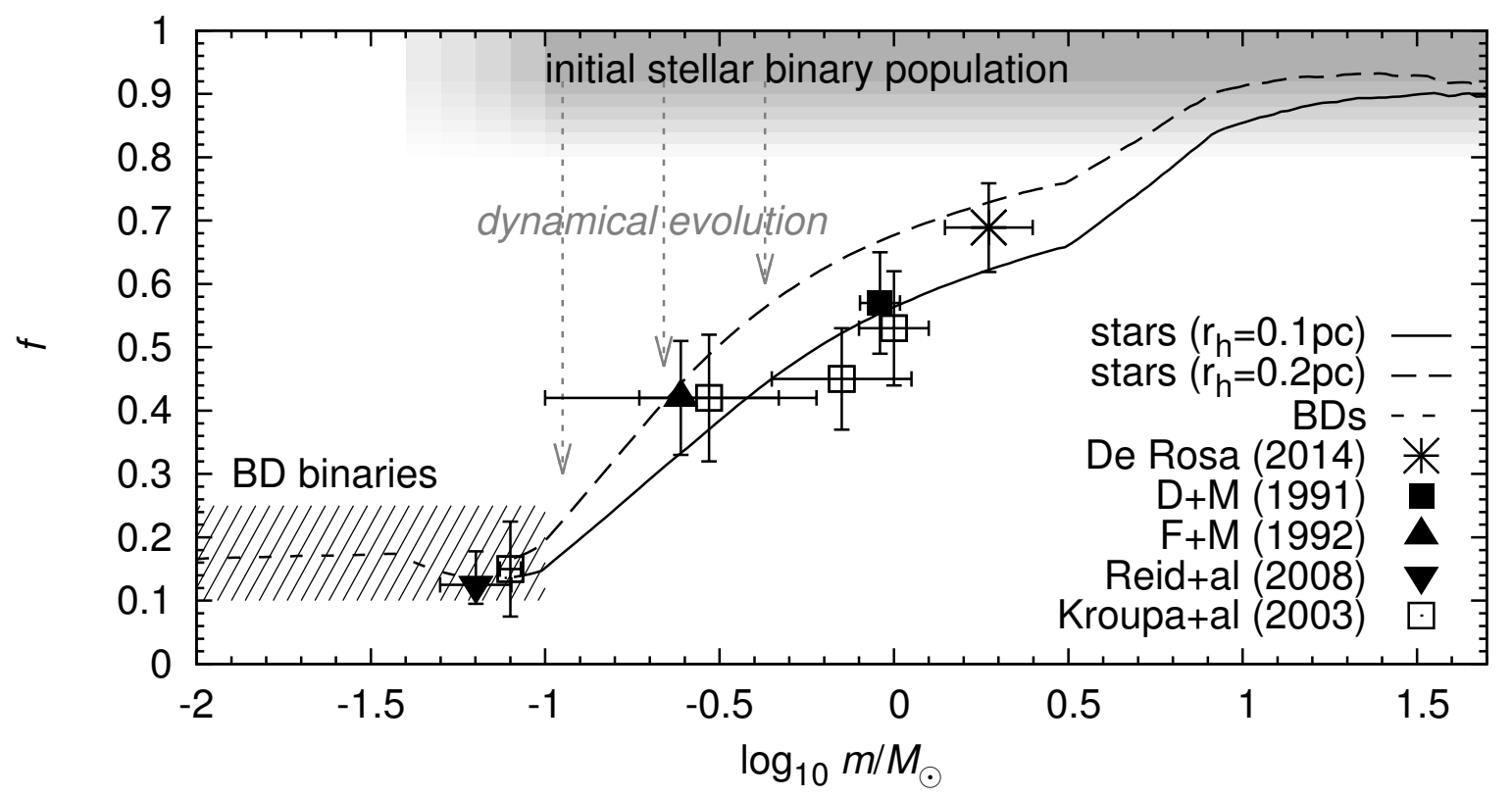

FIG. 6.- Binary fraction for BDs and stars as a function of the primary-object mass. The solid line shows the result of our Monte Carlo computation for stars via DPS pairing for the field population based on the integration of the star-formation outcome over all cluster masses. A half-mass radius $r_{h}=0.1 \mathrm{pc}$ is assumed for all embedded host clusters. The average field-star binary fraction is $f \approx 0.5$ for low-mass to intermediate-mass stars, in accordance with TK07. The long-dashed line refers to the DPS model with $r_{h}=0.2 \mathrm{pc}$, corresponding to a higher binary fraction. The short-dashed curve represents the BD-like population with biased pairing. The single-hatched region in the lower left corner indicates the uncertainty of the substellar binary fraction in the observational data. The smoothly shaded area at the top refers to the initial stellar binary population with a binary fraction of near $100 \%$ (Marks \& Kroupa 2011). Observational field-star data are overplotted for A stars (VAST survey, De Rosa et al.2014 asterisks), G and late-F stars (Duquennoy \& Mayor [1991 filled squares), M to G stars (Kroupa et al. 2003, open squares), M dwarfs (Fischer \& Marcy 1992, upright triangle), and L dwarfs (Reid et al.2008 upside-down triangle).

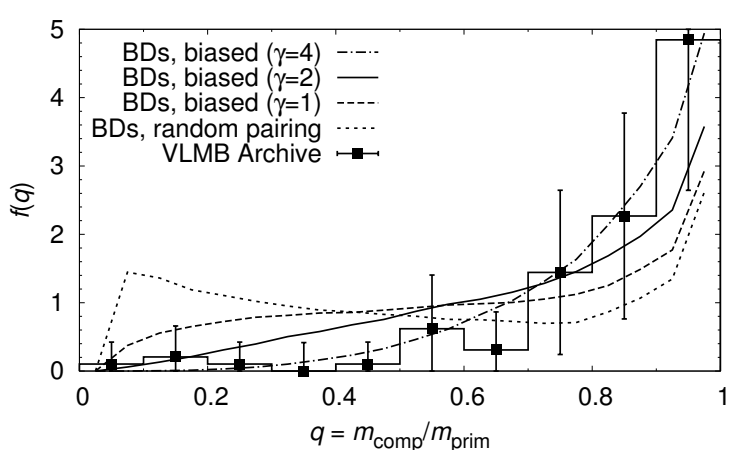

FIG. 7.- Number distribution $f(q)$ for binary BDs between 0.03 and $0.08 M_{\odot}$ as a function of the companion-to-primary mass ratio. The solid line shows the result of our computation for $\gamma=2$, and the dashed and dotted lines refer to the case $\gamma=1$ and random pairing, respectively. For completeness, also the more extreme case of $\gamma=4$ (Duchêne \& Kraus 2013) is also shown as the dash-dotted line. The histogram shows the observational data from the Very-Low-Mass Binary Archive (Burgasser et al. 2007). The peak at $q=1$ is primarily due to the contribution of binaries from the low-mass end of the star-like population because any binary with both components near the lower mass border of a population can only have nearly equal component masses. The area below each curve and below the histogram is normalized to 1 .

This correction term has been identified and quantified here as the RMF which has a similar shape for both analyzed observational reference IMFs by $\mathrm{C} 05$ and TK07. The most striking outcome is a general agreement between the RMF (Equation (1)) as obtained from the theoretical IMF and the observational IMFs, and the mass function of fragments from SPH simulations (Thies et al. 2010). In other words, both PN02 and HC08 IMF models appear to describe essentially the population of stars without a significant fraction of BDs. They can be considered to be successful models of the direct fragmen-

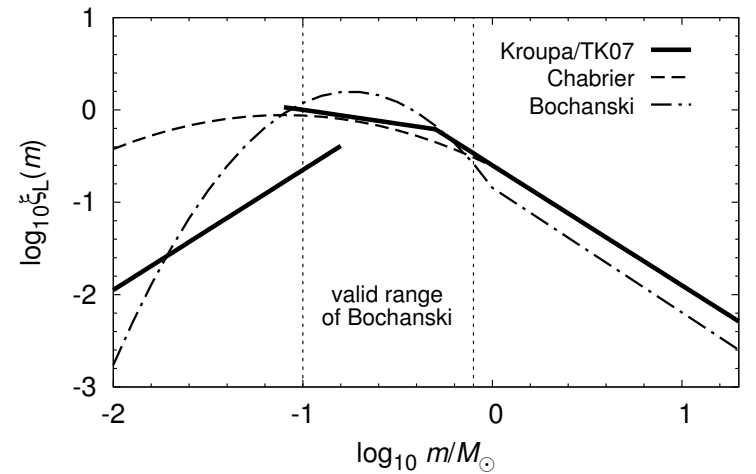

FIG. 8.- Comparison of the Thies \& Kroupa (2007), Chabrier (2005), and the extrapolated Bochanski et al. (2010) IMF, the latter being used by Reggiani \& Meyer (2011). The original Bochanski IMF has been derived from data between 0.1 and $0.8 M_{\odot}$. Note the steeper decline in the Bochanski IMF below its valid mass range relative to the Chabrier IMF.

tation process in molecular clouds. These models cannot, by their nature, accommodate the peripheral fragmentation, e.g., in accretion disks around protostars, which yields the BD-like population (see Section 3.5. The most natural way to explain these results is, to the best of our knowledge, a composite population consisting of a star-like and a BD-like component such that a significant fraction of the BD part comes from an additional process acting during star formation (Reipurth \& Clarke 2001, Stamatellos et al.|2007a, Thies et al.|2010, Basu \& Vorobyov 2012). Essentially this result follows for BDs being less likely to form directly in a star-forming molecular cloud than from dynamically preprocessed material. This is because in order to form only a BD a cloud core needs to have a high pre-collapse density (for it to be gravitationally unsta- 


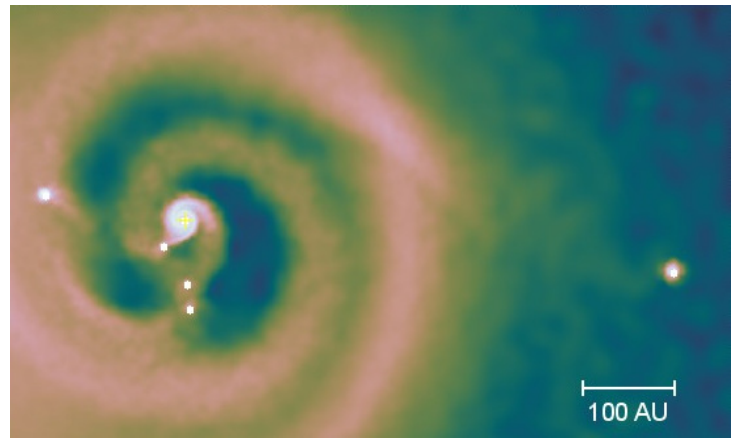

FIG. 9.- Snapshot from SPH model X002 by Thies et al. (2010) showing substellar clumps (with sink particles marked with white points) around a fragmented accretion disk, all of them surrounded by their own accretion envelopes. Note that the escaping object $\left(0.013 M_{\odot}\right.$, i.e. a low-mass BD) to the right has retained its accretion disk (visible as diffuse structure around the sink particle) even after dynamical ejection. The half-mass radius of the envelope is about $10 \mathrm{AU}$, and its mass is $2 \cdot 10^{-4} M_{\odot}$, i.e. almost $2 \%$ of the mas of the escaping object.

ble) while having no further supply of gas in order to limit its further growth in mass. This has already been emphasized by Adams \& Fatuzzo (1996). While there has been a recent discovery of a possible proto-BD in Oph B-11 with a mass of $0.03 M_{\odot}$ (André et al. 2012) its further evolution and possible additional accretion from the surrounding molecular gas remains unclear. And, the existence of the BD-like population formed through peripheral fragmentation does not exclude the formation of BDs via the direct fragmentation channel, i.e. as star-like objects as is evident from the analytical PNO2 and HC08 IMFs.

\subsection{Is There a Separate Substellar Population?}

There is an ongoing discussion whether a separate population is really needed to explain the observed properties. A similar discontinuity between planets and BDs is widely accepted. Chabrier et al. (2014) even report a possible mass overlap of the BD and the giant planet regime as well and suggest a distinction between BDs and planets by their formation history rather than by deuterium burning. This can be seen as an analog to the separate substellar population addressed by our work. Jumper \& Fisher (2013) claim to be able to model the trend toward more tightly bound binaries in the $\mathrm{BD}$ regime. However, because this study already assumes the masses to be drawn from a continuous IMF it a priori excludes the option of a two-component IMF. The AstraLux survey reports a narrower binary separation distribution for M-dwarf binaries compared to binaries with FGK primaries (Janson et al. 2012 and Janson et al. 2014, respectively). Such a narrow peak in the separation distribution may be related to sensitivity artifacts as well as, at least for late-type M dwarfs, due to the mass overlap with the BD-like population that extends up to about $0.2 M_{\odot}$.

In contrast, in a study of dynamical binary evolution Parker \& Goodwin (2011) found that the field populations of $\mathrm{M}$ dwarfs and very-low-mass binaries must reflect very different birth populations, since their dynamical processing is essentially the same.

\subsection{The Brown Dwarf Desert}

Similarly, the BD desert itself has been disputed by several groups who suggest that it is more related to the companion mass ratio rather than to the absolute mass. However, a recent survey by Dieterich et al. (2012) further supports the existence of a true BD desert, i.e. a dearth of substellar companions to primary stars ranging from $M$ to $G$ dwarfs. They found an effective lower mass limit of stellar companions near $0.1 M_{\odot}$ which cannot be related to incompleteness but rather points toward a discontinuity in the pairing statistics close to the hydrogen-burning mass limit. They particularly point out that this discontinuity is essentially independent of the primary star mass. This clearly supports a separate substellar population contributing the majority of BDs and is also in agreement with SPH studies of whole star-forming clouds by, e.g., Bate et al. (2003) and Bate (2009a) who reproduce the formation of BDs largely from fragmenting circumstellar disks. They, however, point out that these simulations tend to overproduce BDs and attribute this to the lack of radiative heating in their model (Bate 2009b, 2012). It should also be noted that Bate (2012) could reproduce a mass function in good agreement with the C05 IMF and that the previous overproduction of BDs was avoided there because the radiative heating reduced the frequency of disk fragmentation.

In a related Monte Carlo study performed here, we also found a good agreement of the two-population composite model with observational data on field very-low-mass stellar and BD binaries (Figure 6). The binarity is well reproduced down to the stellar-substellar border as a continuous function of the primary-star mass in agreement with the observations. More importantly, the mass-ratio distribution of very-low-mass binaries deduced from observational data is well reproduced in our model (Figure 7), once more supporting a bimodal star and BD formation scenario. Whereas starlike binaries are well represented by DPS (Marks et al. 2015), the BD-like binaries apparently do fit a biased pairing where the probability of pairing rises toward more equal component masses following Equation (8). Here, the cases $\gamma=0$ (simple random pairing) and $1 \leq \gamma \leq 2$ (biased toward equal-mass binaries) were compared. The biased pairing case is in better agreement with the observational data. It remains to be studied whether postformation dynamical processing through stellar-dynamical encounters in their birth embedded clusters is responsible for this apparent preference of more equal-mass binaries over more unequal ones in the BD mass range. However, both simple and biased pairing are in reasonable agreement within the uncertainties of the data if a low binary fraction of $10 \%$ within the BD-like population is assumed. A binary fraction of less than $10 \%$ in the theoretical disk fragmentation outcome and the very narrow range of semimajor axes have also been found by Thies et al. (2010), as well as the survival of circumsubstellar accretion disks, as shown in Figure 9. The overall binary fraction for systems within 0.03 and $0.08 M_{\odot}$, irrespective of the population to which they belong, is around $20 \%$ for both simple and biased pairing (i.e. the value chosen for the BD-like population), if the local minimum in the binarity function near the stellar-substellar boundary (Figure 6) is assumed to be an artifact, whereas it is as low as $11 \%$ otherwise. However, observations of VLMSs indicate a binary fraction as low as $12.5 \%$ (Reid et al. 2008), so this "dip" may indeed be real and thus be a result of the overlap of two separate populations.

\subsection{Companion Mass Ratio Distribution}

There is an ongoing discussion whether random pairing is applicable for stars. Reggiani \& Meyer (2011) claim to rule out this model in favor of a universal CMRD. However, their results only cover a narrow range of validity of the underlying 
model IMF (as specified by Bochanski et al. 2010, see Figure 8) that only covers $\mathrm{M}, \mathrm{K}$ and late-G stars, but no BDs. Consequently, the CMRD derived from it is quite limited, especially for M dwarfs, for which no hypothesis test on the pairing rule can be made on this basis. Reggiani \& Meyer (2011) perform pairing experiments that actually exceed the range of validity of the underlying mass function by Bochanski et al. (2010), and therefore their results are not applicable. Goodwin (2013) suggests that a random partition of protostellar cloud fragments is in better agreement with observations of stellar binarity than an initial binary population drawn from the IMF with subsequent dynamical and eigenevolution. Because that study was based on the IMF by Chabrier (2003) without a separate BD treatment there cannot by any satisfactory agreement with the observed substellar CMRD. Even more importantly, the initial binary population needs to be modeled and tested against dynamically barely evolved stellar populations like Taurus-Auriga rather than against the Galactic field. The field population then results from an integration of all dynamically evolved clustered populations following the embedded cluster-mass function (Marks \& Kroupa 2011. Marks et al. 2015).

As a final remark, it is being argued that the existence of disks around young BDs implies that these form like stars supposedly supporting the continuous IMF scenario. Figure 9 is one of many examples where a young BD with an accretion disk formed from peripheral fragmentation in a circumstellar disk is nudged away to become a free-floating BD with a disk.

\section{CONCLUSION}

As the main conclusion from the first part of this study we emphasize the difficulties of theoretical star-formation models to describe both stars and BDs by a single mechanism, namely from direct cloud fragmentation. It remains neces- sary to treat BDs separately which implies a separate albeit related formation channel for the majority of BDs. This already follows from the work of Adams \& Fatuzzo (1996). The theoretical evidence by Stamatellos \& Whitworth (2009), Thies et al. (2010), and Basu \& Vorobyov (2012) supports a separate population by fragmentation of extended young circumstellar disks. If $\mathrm{O}$ stars are close by, the accretion envelope of VLM protostars may be photoevaporated, leaving an unfinished substellar embryo (Kroupa \& Bouvier 2003). In addition, the embryo-ejection model by Reipurth \& Clarke (2001) gives an example of BD formation by ejection of unfinished stellar embryos out of multiple protostar systems. Because these mechanisms are not covered by the analytical cloud fragmentation models by PNO2 and HC08 they do not contribute to the resulting theoretical clump mass function. The same is true for BD formation in dense gaseous filaments that form due to the gravitational pull of surrounding stars (Bonnell et al. 2008) rather than being described by random density fluctuations assumed by PNO2 and C05. Therefore, an analytical model also covering BDs and VLMSs must include such separate formation channels via dynamically preprocessed material (disks, unstable multiple stellar embryo systems, photoevaporation, tidally shaped gas filaments and so on). The normalization may be adjusted empirically or deduced from physical relations based on typical disk-to-stellar mass relations and by the application of turbulence and the Jeans criterion within this preprocessed material. The development of such an analytical or semianalytical model should be the subject of future studies. The observational data thus very strongly suggest that the dominant fraction (Section 3.5) of BDs and VLMSs results from peripheral fragmentation that contributes a separate IMF from that of stars.

This project has been funded by DFG project KR1635/27.

\section{REFERENCES}

Adams, F. C. \& Fatuzzo, M. 1996, ApJ, 464, 256

André, P., Ward-Thompson, D., \& Greaves, J. 2012, Science, 337, 69

Bastian, N., Covey, K. R., \& Meyer, M. R. 2010, ARA\&A, 48, 339

Basu, S., \& Vorobyov, E. I. 2012, ApJ, 750, 30

Bate, M. R. 2009a, MNRAS, 392, 590

-. 2009b, MNRAS, 392, 1363

-. 2012, MNRAS, 419, 3115

Bate, M. R., Bonnell, I. A., \& Bromm, V. 2003, MNRAS, 339, 577

Bochanski, J. J., Hawley, S. L., Covey, K. R., West, A. A., Reid, I. N., Golimowski, D. A., \& Ivezić, Ž. 2010, AJ, 139, 2679

Bonnell, I. A., Clark, P., \& Bate, M. R. 2008, MNRAS, 389, 1556

Bouy, H., Brandner, W., Martín, E. L., Delfosse, X., Allard, F., \& Basri, G. 2003, AJ, 126, 1526

Burgasser, A. J., Kirkpatrick, J. D., Reid, I. N., Brown, M. E., Miskey, C. L., \& Gizis, J. E. 2003, ApJ, 586, 512

Burgasser, A. J., Reid, I. N., Siegler, N., Close, L., Allen, P., Lowrance, P. \& Gizis, J. 2007, in Protostars and Planets V, ed. B. Reipurth, D. Jewitt, \& K. Keil (Univ. Arizona Press, Tucson), 427-441

Chabrier, G. 2003, PASP, 115, 763

Chabrier, G. 2005, in Astrophysics and Space Science Library, Vol. 327, The Initial Mass Function 50 Years Later, ed. E. Corbelli, F. Palla, \& H. Zinnecker, 41

Chabrier, G., Johansen, A., Janson, M., \& Rafikov, R. 2014, in Protostars and Planets VI, ed. H. Beuther, C. Klessen, R. Dullemond, \& T. Henning (Tucson, AZ: University of Arizona Press), TBA

Close, L. M., Siegler, N., Freed, M., \& Biller, B. 2003, ApJ, 587, 407

De Rosa, R. J., et al. 2014, MNRAS, 437, 1216

Dieterich, S. B., Henry, T. J., Golimowski, D. A., Krist, J. E., \& Tanner, A. M. 2012, AJ, 144, 64

Duchêne, G., \& Kraus, A. 2013, ARA\&A, 51, 269

Duquennoy, A., \& Mayor, M. 1991, A\&A, 248, 485

Fischer, D. A., \& Marcy, G. W. 1992, ApJ, 396, 178

Goodwin, S. P. 2013, MNRAS, 430, L6

Goodwin, S. P., \& Kroupa, P. 2005, A\&A, 439, 565

Grether, D., \& Lineweaver, C. H. 2006, ApJ, 640, 1051

Hennebelle, P., \& Chabrier, G. 2008, ApJ, 684, 395

-. 2009, ApJ, 702, 1428
Janson, M., Bergfors, C., Brandner, W., Kudryavtseva, N., Hormuth, F., Hippler, S., \& Henning, T. 2014, ApJ, 789, 102

Janson, M., et al. 2012, ApJ, 754, 44

Joergens, V. 2008, A\&A, 492, 545

Jumper, P. H., \& Fisher, R. T. 2013, ApJ, 769, 9

Kirk, H., \& Myers, P. C. 2012, ApJ, 745, 131

Kraus, A. L., \& Hillenbrand, L. A. 2012, ApJ, 757, 141

Kraus, A. L., White, R. J., \& Hillenbrand, L. A. 2006, ApJ, 649, 306

Kroupa, P. 1995, MNRAS, 277, 1507

-. 2001, MNRAS, 322, 231

-. 2002, Science, 295,82

Kroupa, P., \& Bouvier, J. 2003, MNRAS, 346, 369

Kroupa, P., Bouvier, J., Duchêne, G., \& Moraux, E. 2003, MNRAS, 346, 354

Kroupa, P., Tout, C. A., \& Gilmore, G. 1993, MNRAS, 262, 545

Kroupa, P., Weidner, C., Pflamm-Altenburg, J., Thies, I., Dabringhausen, J., Marks, M., \& Maschberger, T. 2013, in Planets, Stars and Stellar

Systems. Volume 5: Galactic Structure and Stellar Populations, ed. T. D. Oswalt \& G. Gilmore (Springer), 115

Law, N. M., Hodgkin, S. T., \& Mackay, C. D. 2008, MNRAS, 384, 150

Leigh, N. W. C., Mastrobuono-Battisti, A., Perets, H. B., \& Böker, T. 2014 MNRAS, 441, 919

Marks, M., \& Kroupa, P. 2011, MNRAS, 417, 1702

-. 2012, A\&A, 543, A8

Marks, M., Kroupa, P., \& Oh, S. 2011, MNRAS, 417, 1684

Marks, M., Pflamm-Altenburg, J., \& Kroupa, P. 2015, in preparation

Martín, E. L., Barrado y Navascués, D., Baraffe, I., Bouy, H., \& Dahm, S. 2003, ApJ, 594, 525

McCarthy, C., Zuckerman, B., \& Becklin, E. E. 2003, in Brown Dwarfs,

IAU Symposium 211, ed. E. Martín (ASP, San Francisco), 279-+

Padoan, P., \& Nordlund, Å. 2002, ApJ, 576, 870

-. 2004, ApJ, 617, 559

Parker, R. J., \& Goodwin, S. P. 2011, MNRAS, 411, 891

Pflamm-Altenburg, J., Weidner, C., \& Kroupa, P. 2007, ApJ, 671, 1550

Reggiani, M. M., \& Meyer, M. R. 2011, ApJ, 738, 60

Reid, I. N., Cruz, K. L., Burgasser, A. J., \& Liu, M. C. 2008, AJ, 135, 580

Reipurth, B., \& Clarke, C. 2001, AJ, 122, 432 
Reipurth, B., Clarke, C. J., Boss, A. P., et al. 2014, in University of Arizona Space Science Series, Protostars and Planets V, ed. B. Reipurth, D. Jewitt, $\&$ K. Keil (Tucson, AZ: University of Arizona Press)

Stamatellos, D., Hubber, D. A., \& Whitworth, A. P. 2007a, MNRAS, 382, L30

Stamatellos, D., \& Whitworth, A. P. 2009, MNRAS, 392, 413
Stamatellos, D., Whitworth, A. P., Bisbas, T., \& Goodwin, S. 2007b, A\&A, 475,37

Thies, I., \& Kroupa, P. 2007, ApJ, 671, 767

2008, MNRAS, 390,1200

Thies, I., Kroupa, P., Goodwin, S. P., Stamatellos, D., \& Whitworth, A. P 2010, ApJ, 717, 577

Weidner, C., Kroupa, P., \& Bonnell, I. A. D. 2010, MNRAS, 401, 275

Weidner, C., Kroupa, P., \& Pflamm-Altenburg, J. 2013, MNRAS, 434, 84 